\title{
Supersymmetry and Localization in the Quantum Hall Effect
}

\author{
J. Kondev* and J.B. Marston \\ Department of Physics, Brown University, Providence, Rhode Island 02912-1843
}

(February 28, 1997)

cond-mat/9612223

\begin{abstract}
We study the localization transition in the integer quantum Hall effect as described by the network model of quantum percolation. Starting from a path integral representation of transport Green's functions for the network model, which employs both complex (bosonic) and Grassman (fermionic) fields, we map the problem of localization to the problem of diagonalizing a one-dimensional nonHermitian Hamiltonian of interacting bosons and fermions. An exact solution is obtained in a restricted subspace of the Hilbert space which preserves boson-fermion supersymmetry. The physically relevant regime is investigated using the density matrix renormalization group (DMRG) method, and critical behavior is found at the plateau transition.
\end{abstract}

PACS numbers: 71.50.+t, 72.15.Rn, 73.40.Hm

\section{INTRODUCTION}

The physics of electronic transport in the presence of impurities is rich and incompletely understood. Disorder plays a crucial role in the integer quantum Hall effect (IQHE) as it leads to plateaus in the Hall resistance as a function of applied magnetic field. Transitions between plateaus are an especially interesting critical phenomenon. Critical behavior was predicted by Levin, Libby, and Pruisken [1] and was observed experimentally by Wei et al. [2] for temperatures near the critical point at absolute zero, and by Koch et al. [3] for different sample widths. The correlation length exponent $\nu$ was measured to be approximately $\nu \approx 2.3$ and the dynamical exponent $z$ was found to be equal to unity.

Progress toward a theoretical understanding of the plateau transition was made with the introduction of a quantum tunneling network model by Chalker and Coddington [4]. Subsequent numerical studies [5] of the Chalker-Coddington network model yielded values for $\nu$ consistent with experiment. An argument based on the semi-classical picture of electron motion in the IQHE suggests that the value of $\nu$ is $7 / 3$ [6], but so far analytical derivations are still unconvincing. Lee [7] mapped the network model to the $n \rightarrow 0$ replica limit of a one-dimensional quantum $S U(2 n)$ Heisenberg antiferromagnet, where the plateau transition is equivalent to a transition between two dimerized phases. Lee and Wang [8] extended this mapping to the replica limit of an associated Hubbard model.

In a continuum picture Ludwig et al. [9] mapped the IQHE problem to a model of two-dimensional Dirac fermions interacting via random scalar and gauge fields. They speculated that the fixed point controlling the plateau transitions was at strong-coupling and thus not directly accessible by methods of weak-coupling perturbation theory or bosonization.

There is another field theory method besides replication which permits the averaging over quenched disorder: supersymmetry. McKane showed that the introduction of equal numbers of bosonic and fermionic degrees of freedom made the partition function independent of the particular realization of disorder. Disorder-averaged observables then could be expressed as the Green's functions of a field theory [10]. Efetov used this idea to reformulate the $\sigma$-model approach to Anderson localization [11] and Affleck, in a saddle-point calculation [12], showed that the density of states in a uniform magnetic field and a white noise potential was non-vanishing. Zirnbauer [13] applied the method to transport properties and derived both the supersymmetric $\sigma$-model and an antiferromagnetic supersymmetric spinchain. Balents, Fisher and Zirnbauer [14 recently applied this approach to the simpler problem of the chiral metal and exactly solved the resulting ferromagnetic supersymmetric quantum spin-chain. Magnon excitations were identified as the expected diffusive modes.

In contrast to the supersymmetric ferromagnet [14,15], little is known about the antiferromagnetic supersymmetric spin chain. In this paper we rederive it in terms familiar to condensed matter physicists and find an exact analytic solution in a particular limit of the model, which opens up the possibility of studying the plateau transition perturbatively. We also examine the chain in the physically relevant limit by means of the Density Matrix Renormalization

*jane@barus.physics.brown.edu 
Group (DMRG) method and demonstrate the existence of a critical point. The correlation length exponent that we find is consistent with previous numerical studies of the Chalker-Coddington network model.

\section{NETWORK MODEL}

To describe the transition between quantum Hall plateaus Chalker and Coddington introduced a lattice model that combines the two most important features of electronic transport in a magnetic field and in the presence of disorder: percolation and quantum tunneling. The model consists of a network of tunneling centers which form a square lattice in the $\mathrm{x}$-y plane; Fig. 11(a). The electron wavefunction is represented by complex amplitudes $\mathrm{z}_{i}$ which are defined at the bond centers. In the semiclassical picture of electron transport in the IQHE [16] the guiding center of the electron's orbit propagates along a contour line of the random potential. At the saddle points, where two contours pass within a cyclotron radius, the electron can tunnel. Due to the presence of a perpendicular magnetic field the electron acquires a random Aharonov-Bohm phase between two tunneling events. In the network model this phase is represented by random scattering matrices $(\mathrm{S})$ that relate the amplitudes of the electron wavefunction on the outgoing bonds to the amplitudes on the incoming bonds, Fig. 1 (a). The scattering matrices are unitary and are typically chosen to be independently Gaussian distributed.
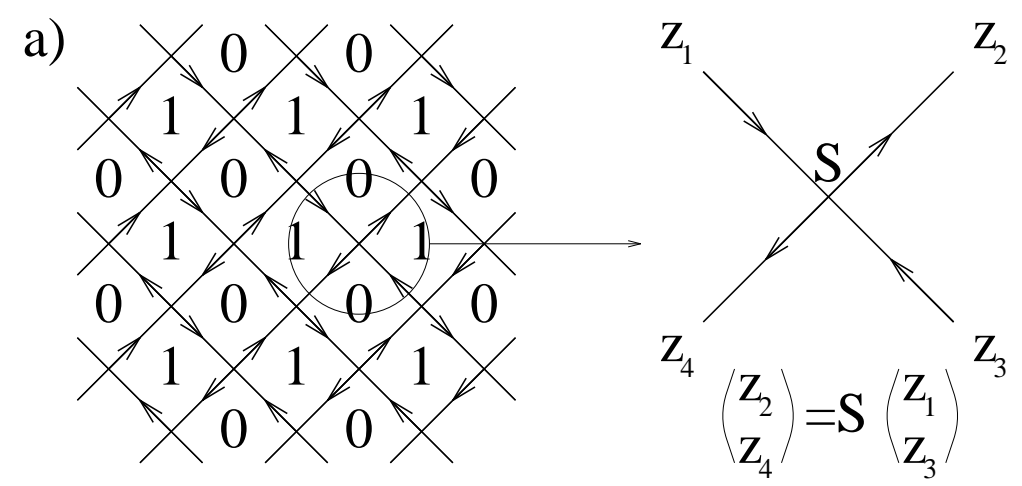

b)

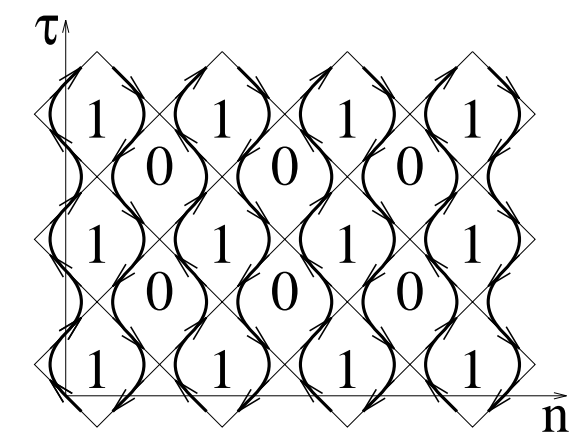

FIG. 1. (a) The Chalker-Coddington network model. Amplitudes of the electron wavefunction ( $\left.\mathrm{z}_{i}\right)$ are assigned to bonds, while the random scattering matrices $(\mathrm{S})$ are assigned to vertices of the network. 1 and 0 denote Hall droplets associated with two consecutive plateaus. (b) The Hamiltonian representation of the network model with counterpropagating chiral fermions as the edge states of the Hall droplets; the tunneling between the edge states is random.

An alternative description of the network model was recently proposed by Lee [7] where the random scattering matrices are replaced by a Hamiltonian which describes a one-dimensional array of counterpropagating edge states in the presence of disorder, as shown in Fig. 1 (b). The direction of propagation will be later interpreted as the imaginary time; to emphasize this point of view we relabel the $y$-coordinate as $\tau$ and also denote the $x$-coordinate with the integer index $n$.

Expressed in terms of electron creation $\hat{\psi}^{\dagger}$ and annihilation $\hat{\psi}$ operators the Hamiltonian may be written as

$$
H\left[\hat{\psi}^{\dagger}, \hat{\psi}\right]=\sum_{n, \tau}\left[(-1)^{n} \hat{\psi}_{n, \tau}^{\dagger}\left(\mathrm{i} \partial_{\tau}+w_{n, \tau}\right) \hat{\psi}_{n, \tau}-\left(t_{n, \tau} \hat{\psi}_{n, \tau}^{\dagger} \hat{\psi}_{n+1, \tau}+t_{n, \tau}^{*} \hat{\psi}_{n+1, \tau}^{\dagger} \hat{\psi}_{n, \tau}\right)\right]
$$

where the sum is over the discrete vertices of the network model, and $\hat{\psi}_{n, \tau}^{\dagger}\left(\mathrm{i} \partial_{\tau}\right) \hat{\psi}_{n, \tau}$ is defined in momentum (Fourier) space, in the limit of small $k_{\tau}$, as $\hat{\psi}_{n, k_{\tau}}^{\dagger}\left(k_{\tau}\right) \hat{\psi}_{n, k_{\tau}}$. To avoid ordering ambiguities, for now we keep $\tau$ discrete and refrain 
from taking the continuum limit until later, after the average over disorder has been performed. The Hamiltonian, Eq. 14. reflects the chiral nature and linear dispersion of the edge states, with alternating propagation forward and backward in the $\tau$ direction at Fermi velocities \pm 1 . It also incorporates complex tunneling amplitudes $t_{n, \tau}$ with random phases, which represent the random Aharonov-Bohm phases of the electrons discussed above, as well as random on-site potentials $w_{n, \tau}$. The random terms are taken to be independently Gaussian distributed with zero mean, and variance

$$
\begin{aligned}
\ll w_{n, \tau} w_{n^{\prime}, \tau^{\prime}} \gg & =2 U \delta_{n, n^{\prime}} \delta_{\tau, \tau^{\prime}} \\
\ll t_{n, \tau}^{*} t_{n^{\prime}, \tau^{\prime}} \gg & =J_{n} \delta_{n, n^{\prime}} \delta_{\tau, \tau^{\prime}}, \quad J_{n}=J \times\left(1+(-1)^{n} R\right) .
\end{aligned}
$$

Staggered modulation in the parameter $J_{n}$ accounts for differences in tunneling between counterpropagating electrons in adjacent plaquette-columns as depicted in Fig. 1 (b). As $R \rightarrow-1$ electrons only circulate clockwise around the 1 plaquettes. We can picture these plaquettes as isolated lakes of electrons (regions of attractive potential with filling fraction 1) separated by dry land (repulsive potential and filling fraction 0 ). In the opposite limit $R \rightarrow 1$ electrons circulate counterclockwise only around 0 plaquettes, which corresponds to isolated islands (empty regions of filling fraction 0 ) in the midst of an ocean of (filling fraction 1) electrons. Precisely at $R=0$ the land and sea cover equal areas and we expect critical quantum percolation associated with the transition between the 0 and 1 plateau. The percolating edge states are characterized by a diverging length scale, $\xi \sim R^{-\nu}$. Critical behavior in accord with this scenario was found from the disorder averaged transport Green's function of the Hamiltonian (Eq. 11) by Wang and Lee [8]. From a Monte-Carlo treatment of the replicated Hamiltonian they obtained a correlation length exponent $\nu=2.33 \pm 0.03$, in good agreement with experiments and other numerical simulations of the network model.

\section{TRANSPORT GREEN'S FUNCTIONS}

To study the localization-delocalization transition in the network model as described by the random Hamiltonian, Eq. 1, we focus on the disorder-averaged transport Green's function [17]

$$
K(1,2)=\ll G_{+}(1,2) G_{-}(2,1) \gg
$$

where

$$
G_{ \pm}(1,2)=\left\langle 1\left|\frac{1}{H-E \pm \mathrm{i} \eta}\right| 2\right\rangle
$$

are the advanced and retarded Green's functions; $E$ is the energy and $\eta$ a positive infinitesimal. We introduce the usual shorthand for coordinates with $1 \equiv\left(n_{1}, \tau_{1}\right)$ and $2 \equiv\left(n_{2}, \tau_{2}\right)$. In the localized regime, $R$ is non-zero, and we expect the transport Green's function to be short-ranged

$$
K(1,2) \sim \exp \left(-r_{12} / \xi\right)
$$

where $r_{12}$ is the distance between points 1 and 2 , and $\xi \sim R^{-\nu}$. The correlation length $\xi$ reflects the finite extent of the electron wavefunction. To calculate the matrix elements of the resolvent of $H$ appearing in the expression for the advanced and retarded Green's functions, Eq. 1, we introduce a pair of complex scalar fields

$$
\phi(n, \tau)=\left(\begin{array}{c}
\phi_{\uparrow}(n, \tau) \\
\phi_{\downarrow}(n, \tau)
\end{array}\right) .
$$

The Green's functions [17] can now be written as

$$
\begin{aligned}
& G_{+}(1,2)=-\mathrm{i}\left\langle\phi_{\uparrow}(1) \phi_{\uparrow}^{*}(2)\right\rangle \\
& G_{-}(1,2)=+\mathrm{i}\left\langle\phi_{\downarrow}(1) \phi_{\downarrow}^{*}(2)\right\rangle,
\end{aligned}
$$

where averages of observables $\mathcal{O}$ are taken with respect to a Gaussian measure,

$$
\begin{aligned}
\langle\mathcal{O}\rangle & =\mathcal{Z}^{-1} \int \mathrm{D} \phi \mathrm{D} \phi^{*} \mathcal{O}\left[\boldsymbol{\phi}, \phi^{*}\right] \exp \left(-S\left[\boldsymbol{\phi}, \boldsymbol{\phi}^{*}\right]\right) \\
S\left[\phi, \phi^{*}\right] & =-\mathrm{i}\left\{H\left[\phi_{\uparrow}\right]+\mathrm{i} \eta \sum_{n, \tau} \phi_{\uparrow}(n, \tau) \phi_{\uparrow}^{*}(n, \tau)\right\}+\mathrm{i}\left\{H\left[\phi_{\downarrow}\right]-\mathrm{i} \eta \sum_{n, \tau} \phi_{\downarrow}(n, \tau) \phi_{\downarrow}^{*}(n, \tau)\right\} .
\end{aligned}
$$


In the above equation we have set $E=0$; in the advanced and retarded Green's functions non-zero energy is easily recovered by replacing $\eta$ with $\eta \pm \mathrm{i} E$. The choice of signs in the terms proportional to $\eta$ is dictated by the requirement that the Gaussian integrals be convergent for $\eta>0$. The quadratic form $H[\phi]$ is obtained simply by replacing the operators $\hat{\psi}_{n, \tau}$ and $\hat{\psi}_{n, \tau}^{\dagger}$ in the Hamiltonian of the network model, Eq. 11, with complex scalar fields $\phi(n, \tau)$ and $\phi^{*}(n, \tau)$. Finally, the normalization factor

$$
\mathcal{Z}=\int \mathrm{D} \phi \mathrm{D} \phi^{*} \exp \left(-S\left[\phi, \phi^{*}\right]\right)
$$

can be written as Gaussian integral over Grassman fields 18]

$$
\mathcal{Z}^{-1}=\int \mathrm{D} \boldsymbol{\psi} \mathrm{D} \overline{\boldsymbol{\psi}} \exp (-S[\boldsymbol{\psi}, \overline{\boldsymbol{\psi}}])
$$

where the anticommuting fields

$$
\boldsymbol{\psi}(n, \tau)=\left(\begin{array}{c}
\psi_{\uparrow}(n, \tau) \\
\psi_{\downarrow}(n, \tau)
\end{array}\right)
$$

are the supersymmetric partners of the scalar fields. Using both Eqs. 10 and 8, the correlation functions, Eq. 7, may be written as a combined Gaussian integral over scalar and Grassman fields

$$
\langle\mathcal{O}\rangle=\int \mathrm{D} \phi \mathrm{D} \phi^{*} \mathrm{D} \boldsymbol{\psi} \mathrm{D} \overline{\boldsymbol{\psi}} \mathcal{O}\left[\boldsymbol{\phi}, \boldsymbol{\phi}^{*}\right] \exp \left(-S\left[\boldsymbol{\phi}, \boldsymbol{\phi}^{*}\right]-S[\boldsymbol{\psi}, \overline{\boldsymbol{\psi}}]\right) .
$$

The utility of this representation lies in the fact that, for each realization of disorder, the normalization factor has been absorbed into the action. Now the average over disorder can be interchanged with integration over the scalar and Grassman fields, and the problem of computing the disorder-averaged transport Green's function, Eq. 3, reduces to the problem of calculating correlation functions in a pure field theory, albeit one with interacting fermionic and bosonic degrees of freedom. Our strategy in what follows will be to perform the disorder average in the path integral, Eq. 12. Then, because the $\tau$-derivative term is linear, the disorder-averaged path integral will be interpreted as a coherent state path integral for a one-dimensional Hamiltonian. The problem of calculating disorder-averaged correlation functions for the network model which is infinite in the $\tau$ direction is thus transformed to the problem of computing expectation values in the ground state of this Hamiltonian.

\section{NON-HERMITIAN HAMILTONIAN}

The supersymmetry method, as discussed in the preceding section, allows us to perform the average over disorder inside the path integral. Before proceeding with this step we first transform the scalar and Grassman fields on even sites,

n even:

$$
\phi_{\downarrow}(n, \tau) \rightarrow \phi_{\downarrow}^{*}(n, \tau) \quad \psi_{\downarrow}(n, \tau) \rightarrow-\bar{\psi}_{\downarrow}(n, \tau)
$$

$$
\phi_{\downarrow}^{*}(n, \tau) \rightarrow \phi_{\downarrow}(n, \tau) \quad \bar{\psi}_{\downarrow}(n, \tau) \rightarrow \psi_{\downarrow}(n, \tau)
$$

and on odd sites

n odd:

$$
\phi_{\uparrow}(n, \tau) \rightarrow \phi_{\uparrow}^{*}(n, \tau) \quad \psi_{\uparrow}(n, \tau) \rightarrow-\bar{\psi}_{\uparrow}(n, \tau)
$$

$$
\phi_{\uparrow}^{*}(n, \tau) \rightarrow \phi_{\uparrow}(n, \tau) \quad \bar{\psi}_{\uparrow}(n, \tau) \rightarrow \psi_{\uparrow}(n, \tau) ;
$$

the remaining fields are left unchanged. The transformation is guided by two requirements. First, the linear derivative term in the action acquires the canonical form for both scalar and Grassman fields [18],

$$
S_{\tau}=\sum_{n, \tau}\left[\overline{\boldsymbol{\psi}}(n, \tau) \cdot \partial_{\tau} \boldsymbol{\psi}(n, \tau)+\boldsymbol{\phi}^{*}(n, \tau) \cdot \partial_{\tau} \phi(n, \tau)\right]
$$

Second, the sign of the $\eta$ term in the bosonic part of the action must be such to ensure convergence of the path integral; see Eq. 18 below. 
Next we perform the disorder average inside the path integral using Eq. 2:

$$
\ll \exp \left(-S\left[\boldsymbol{\phi}, \boldsymbol{\phi}^{*}\right]-S[\boldsymbol{\psi}, \overline{\boldsymbol{\psi}}]\right) \gg=\exp \left(-S_{\mathrm{SUSY}}\left[\boldsymbol{\phi}, \boldsymbol{\phi}^{*}, \boldsymbol{\psi}, \overline{\boldsymbol{\psi}}\right]\right) .
$$

The action $S_{\mathrm{SUSY}}$ can be written as a sum of three terms

$$
S_{\mathrm{SUSY}}=S_{\tau}+S_{U}+S_{J}+S_{\eta}
$$

where $S_{\tau}$ is given by Eq. 15, and

$$
\begin{aligned}
S_{U} & =U \sum_{n, \tau}\left[\overline{\boldsymbol{\psi}}(n, \tau) \sigma_{z} \boldsymbol{\psi}(n, \tau)+\boldsymbol{\phi}^{*}(n, \tau) \sigma_{z} \boldsymbol{\phi}(n, \tau)\right]^{2} \\
S_{J} & =\sum_{n, \tau} J_{n}\left[(-1)^{n+1} \mathcal{C}(n, \tau)+\mathcal{B}(n, \tau)\right]\left[(-1)^{n} \overline{\mathcal{C}}(n, \tau)+\mathcal{B}^{*}(n, \tau)\right] \\
S_{\eta} & =\eta \sum_{n, \tau}\left[\overline{\boldsymbol{\psi}}(n, \tau) \cdot \boldsymbol{\psi}(n, \tau)+\boldsymbol{\phi}^{*}(n, \tau) \cdot \boldsymbol{\phi}(n, \tau)\right]
\end{aligned}
$$

$\sigma_{z}=\operatorname{diag}(1,-1)$ is the $2 \times 2$ Pauli matrix operating in spin space $(\uparrow, \downarrow)$, and we have introduced new fields bilinear in the original Grassman and scalar fields which live on the bonds,

$$
\begin{aligned}
& \mathcal{B}(n, \tau)=\phi_{\uparrow}^{*}(n, \tau) \phi_{\uparrow}^{*}(n+1, \tau)-\phi_{\downarrow}(n, \tau) \phi_{\downarrow}(n+1, \tau) \\
& \mathcal{C}(n, \tau)=\bar{\psi}_{\uparrow}(n, \tau) \bar{\psi}_{\uparrow}(n+1, \tau)+\psi_{\downarrow}(n, \tau) \psi_{\downarrow}(n+1, \tau) .
\end{aligned}
$$

Now it is safe to take the $\tau$-continuum limit and interpret the $\tau$ direction as imaginary time. The action $S_{\mathrm{SUSY}}$ is precisely that which appears in a coherent state path integral representation of the partition function for a onedimensional Hamiltonian given by:

$$
H_{\mathrm{SUSY}}=H_{U}+H_{J}+H_{\eta} .
$$

The different terms in the Hamiltonian can be read off from the corresponding terms in $S_{\mathrm{SUSY}}$ only after the scalar and Grassman fields have been properly ordered: the fields are commuted and anticommuted so that $\phi^{*}$ and $\bar{\psi}$ always appear before $\phi$ and $\psi$. The normal ordering is essential [18] because the scalar and Grassman fields appear in the coherent state path integral as left and right eigenvalues of creation and annihilation operators, respectively. Introducing bosonic operators which satisfy the usual canonical commutation relations $\left[\hat{b}_{\uparrow}, \hat{b}_{\uparrow}^{\dagger}\right]=\left[\hat{b}_{\downarrow}, \hat{b}_{\downarrow}^{\dagger}\right]=1$ and fermionic operators which satisfy canonical anticommutation relations $\left\{\hat{c}_{\uparrow}, \hat{c}_{\uparrow}^{\dagger}\right\}=\left\{\hat{c}_{\downarrow}, \hat{c}_{\downarrow}^{\dagger}\right\}=1$, normal ordering, and dropping the hat over the operators for clarity, we obtain

$$
\begin{aligned}
& H_{U}=U \sum_{n}\left[\left(\mathcal{N}_{\uparrow n}^{c}-\mathcal{N}_{\downarrow n}^{c}+\mathcal{N}_{\uparrow n}^{b}-\mathcal{N}_{\downarrow n}^{b}\right)^{2}-\left(\mathcal{N}_{\uparrow n}^{c}+\mathcal{N}_{\downarrow n}^{c}+\mathcal{N}_{\uparrow n}^{b}+\mathcal{N}_{\downarrow n}^{b}\right)\right] \\
& H_{J}=\sum_{n} J_{n}\left[: \mathcal{B}_{n} \mathcal{B}_{n}^{\dagger}:-: \mathcal{C}_{n} \mathcal{C}_{n}^{\dagger}:+(-1)^{n+1}\left(\mathcal{C}_{n} \mathcal{B}_{n}^{\dagger}-\mathcal{C}_{n}^{\dagger} \mathcal{B}_{n}\right)\right] \\
& H_{\eta}=\eta \sum_{n}\left[\mathcal{N}_{\uparrow n}^{b}+\mathcal{N}_{\downarrow n}^{b}+\mathcal{N}_{\uparrow n}^{c}+\mathcal{N}_{\downarrow n}^{c}\right] .
\end{aligned}
$$

We have introduced the boson and fermion number operators $\mathcal{N}_{\uparrow}^{b}=b_{\uparrow}^{\dagger} b_{\uparrow}, \mathcal{N}_{\downarrow}^{b}=b_{\downarrow}^{\dagger} b_{\downarrow}, \mathcal{N}_{\uparrow}^{c}=c_{\uparrow}^{\dagger} c_{\uparrow}$, and $\mathcal{N}_{\downarrow}^{c}=c_{\downarrow}^{\dagger} c_{\downarrow}$. The remaining bilinear operators take their form from Eq. 19:

$$
\begin{aligned}
& \mathcal{B}_{n}=b_{\uparrow n}^{\dagger} b_{\uparrow n+1}^{\dagger}-b_{\downarrow n} b_{\downarrow n+1} \\
& \mathcal{C}_{n}=c_{\uparrow n}^{\dagger} c_{\uparrow n+1}^{\dagger}+c_{\downarrow n} c_{\downarrow n+1},
\end{aligned}
$$

and products of these operators which appear in $H_{J}$ are normal ordered. The terms $H_{U}$ and $H_{\eta}$ are Hermitian while $H_{J}$ is not. The lack of Hermiticity has its origin in our treatment of one of the spatial directions as the time direction. Evolution forward in real time must be unitary, but no such restriction holds for evolution in a spatial direction. 


\section{EXACTLY SOLVABLE LIMIT}

The ground state of $H_{\text {SUSY }}$ holds the key to understanding the localization transition in the network model. The disorder-averaged transport Green's function can be written as a ground state average,

$$
K(1,2)=\left\langle c_{\uparrow}^{\dagger}(1) c_{\uparrow}^{\dagger}(2) c_{\downarrow}^{\dagger}(2) c_{\downarrow}^{\dagger}(1)\right\rangle_{\mathrm{SUSY}}
$$

if 1 is an odd lattice site and 2 is an even site. This equation follows from Eqs. 3 and 7 , once the transformations in Eqs. 13 and 14 have been taken into account, and after the Grassman fields have been replaced with the fermion creation and annihilation operators in the Heisenberg representation. To find the ground state we first perform a canonical transformation

$$
\begin{array}{ll}
c_{\downarrow} \rightarrow c_{\downarrow}^{\dagger} & b_{\downarrow} \rightarrow \bar{b}_{\downarrow} \equiv b_{\downarrow}^{\dagger} \\
c_{\downarrow}^{\dagger} \rightarrow c_{\downarrow} & b_{\downarrow}^{\dagger} \rightarrow-b_{\downarrow}
\end{array}
$$

on every site of the chain. This transformation preserves the canonical commutation and anticommutation relations between creation and annihilation operators, and it ensures that $H_{\text {SUSY }}$ commutes with the number operator $\mathcal{N}_{n}=$ $\mathcal{N}_{\uparrow n}^{c}+\mathcal{N}_{\downarrow n}^{c}+\mathcal{N}_{\uparrow n}^{b}+\mathcal{N}_{\downarrow n}^{b}$ on each site, where the new $\mathcal{N}_{\downarrow}^{b}=\bar{b}_{\downarrow} b_{\downarrow}$. Note, however, that in the bosonic sector the new $b_{\downarrow}$ and $\bar{b}_{\downarrow}$ are not Hermitian conjugates of each other, rather, $\left(\bar{b}_{\downarrow}\right)^{\dagger}=-b_{\downarrow}$. The transformation also removes the explicit $S U(2)$ spin symmetry as the $\uparrow$-spin operators are unchanged.

As $\left[H_{\mathrm{SUSY}}, \mathcal{N}_{n}\right]=0$ the Hilbert space breaks up into sectors with different numbers of particles on each site, and we are faced with the task of identifying the lowest energy sector. After performing the canonical transformation, Eq. 24, the new $H_{U}$ term is

$$
H_{U}=U \sum_{n}\left[\left(\mathcal{N}_{\uparrow n}^{b}+\mathcal{N}_{\downarrow n}^{b}+\mathcal{N}_{\uparrow n}^{c}+\mathcal{N}_{\downarrow n}^{c}\right)^{2}-\left(\mathcal{N}_{\uparrow n}^{b}-\mathcal{N}_{\downarrow n}^{b}+\mathcal{N}_{\uparrow n}^{c}-\mathcal{N}_{\downarrow n}^{c}\right)\right] .
$$

For large $U$ the ground state lies in the subspace of the total Hilbert space which has either no particles, or just one $\uparrow$-fermion or one $\uparrow$-boson per site, as there is a relative energy cost of $2 U$ for a $\downarrow$-particle. The energy of the vacuum state, however, is raised with respect to the singly-occupied states once the other two terms in the Hamiltonian, Eq. 21 are included. As the ground state occupancy is thus non-zero, the density of states (DOS) is also non-zero. This is as expected because the DOS does not vanish either at the Anderson transition 17] or in the Landau level problem [12].

For large $U$ the supersymmetric exchange Hamiltonian $\left(H_{J}\right)$ can be written as

$$
H_{J}=\sum_{n} J_{n}\left[\mathcal{N}_{\uparrow n}^{b} \mathcal{N}_{\uparrow n+1}^{b}-\mathcal{N}_{\uparrow n}^{c} \mathcal{N}_{\uparrow n+1}^{c}+(-1)^{n+1}\left(c_{\uparrow n}^{\dagger} b_{\uparrow n} c_{\uparrow n+1}^{\dagger} b_{\uparrow n+1}+b_{\uparrow n}^{\dagger} c_{\uparrow n} b_{\uparrow n+1}^{\dagger} c_{\uparrow n+1}\right)\right] ;
$$

all the terms with $\downarrow$-spin fermions or bosons have been eliminated. Physically this simply means that there is no transport in the $U \gg J$ limit.

The large- $U$ Hamiltonian, Eq. 26, is non-Hermitian, degenerate, and it is also defective: its eigenstates do not span the Hilbert space. The defect in $\vec{H}_{J}$ is evident even for just two sites with a four-dimensional Hilbert space spanned by: $\left\{b_{\uparrow 1}^{\dagger} b_{\uparrow 2}^{\dagger}|0\rangle, b_{\uparrow 1}^{\dagger} c_{\uparrow 2}^{\dagger}|0\rangle, c_{\uparrow 1}^{\dagger} b_{\uparrow 2}^{\dagger}|0\rangle, c_{\uparrow 1}^{\dagger} c_{\uparrow 2}^{\dagger}|0\rangle\right\}$. Only three (not four) distinct eigenstates of $H_{J}$ can be constructed. Dropping the spin index for clarity, and setting $J_{n}=1$, the two site Hamiltonian may be written:

$$
H_{J}=b_{2}^{\dagger} b_{2} b_{1}^{\dagger} b_{1}-c_{2}^{\dagger} c_{2} c_{1}^{\dagger} c_{1}-c_{2}^{\dagger} c_{1}^{\dagger} b_{1} b_{2}+b_{2}^{\dagger} b_{1}^{\dagger} c_{1} c_{2} \text {. }
$$

As the Hamiltonian is non-symmetric, there are both right eigenstates:

$$
H_{J}\left|\Psi^{R}(a)\right\rangle=\epsilon_{a}\left|\Psi^{R}(a)\right\rangle
$$

and left eigenstates:

$$
\left\langle\Psi^{L}(a)\right| H_{J}=\left\langle\Psi^{L}(a)\right| \epsilon_{a},
$$

with $\left\langle\Psi^{L}(a)\right| \equiv\left(\left|\Psi^{L}(a)\right\rangle\right)^{\dagger} \neq\left\langle\Psi^{R}(a)\right|$ in general. In this case the three eigenstates are degenerate, with zero energy. The right eigenstates are:

$$
\begin{aligned}
& \left|\Psi^{R}(1)\right\rangle=c_{1}^{\dagger} b_{2}^{\dagger}|0\rangle \\
& \left|\Psi^{R}(2)\right\rangle=b_{1}^{\dagger} c_{2}^{\dagger}|0\rangle \\
& \left|\Psi^{R}(3)\right\rangle=\left(b_{1}^{\dagger} b_{2}^{\dagger}+c_{1}^{\dagger} c_{2}^{\dagger}\right)|0\rangle
\end{aligned}
$$


as may be easily verified by direct substitution into Eq. 28. The defect may be removed with the addition of a small, supersymmetry breaking, chemical potential for the fermions:

$$
H_{J} \rightarrow H_{J}+\mu_{c} N_{c} ; \mu_{c}<0
$$

where $N_{c} \equiv \sum_{n}\left(\mathcal{N}_{\uparrow n}^{c}+\mathcal{N}_{\downarrow n}^{c}\right)$ is the total fermion number operator. This term lifts some of the degeneracy and splits the third state into two distinct ones:

$$
\left|\Psi^{R}(3 \pm)\right\rangle=\frac{1}{\sqrt{N}}\left[\left(-\mu_{c} \pm \sqrt{\mu_{c}^{2}-2 \mu_{c}}\right) b_{1}^{\dagger} b_{2}^{\dagger}+\left(\mu_{c} \pm \sqrt{\mu_{c}^{2}-2 \mu_{c}}\right) c_{1}^{\dagger} c_{2}^{\dagger}\right]|0\rangle
$$

with energies $\epsilon_{3 \pm}=\mu_{c} \pm \sqrt{\mu_{c}^{2}-2 \mu_{c}} ; N$ is the normalization constant. The leading dependence of the energies on $\sqrt{\mu_{c}}$, as opposed to linear dependence on $\mu_{c}$, is a telltale sign of the defectiveness of the Hamiltonian in the $\mu_{c} \rightarrow 0$ limit. The left eigenstates are easily found:

$$
\begin{aligned}
\left\langle\Psi^{L}(1)\right| & =\langle 0| b_{2} c_{1} \\
\left\langle\Psi^{L}(2)\right| & =\langle 0| c_{2} b_{1} \\
\left\langle\Psi^{L}(3 \pm)\right| & =\langle 0|\left[\left(-\mu_{c} \pm \sqrt{\mu_{c}^{2}-2 \mu_{c}}\right) b_{2} b_{1}-\left(\mu_{c} \pm \sqrt{\mu_{c}^{2}-2 \mu_{c}}\right) c_{2} c_{1}\right] \frac{ \pm 1}{\sqrt{N}}
\end{aligned}
$$

Now the four states can be properly normalized,

$$
\left\langle\Psi^{L}(b) \mid \Psi^{R}(a)\right\rangle=\delta_{a b},
$$

by setting $N=-4 \mu_{c} \sqrt{\mu_{c}^{2}-2 \mu_{c}}$.

Before turning to the problem of more than two sites, we examine the eigenstates of $H_{\text {SUSY }}$ with $\downarrow$-spin excitations in the physical, finite- $U$ limit. By direct examination of the Hamiltonian we find that eight right-eigenstates can be formed by placing one $\uparrow$-spin and one $\downarrow$-spin particle on the two sites:

$$
b_{\uparrow 1}^{\dagger} c_{\downarrow 2}^{\dagger}|0\rangle, \quad b_{\uparrow 1}^{\dagger} b_{\downarrow 2}^{\dagger}|0\rangle ;
$$

the other six are obtained by exchanging $b$ with $c$ or $\uparrow$ with $\downarrow$. Two more eigenstates with two $\downarrow$-spin excitations are

$$
c_{\downarrow 1}^{\dagger} b_{\downarrow 2}^{\dagger}|0\rangle, \quad b_{\downarrow 1}^{\dagger} c_{\downarrow 2}^{\dagger}|0\rangle .
$$

These ten states, as well as two other states which are not as simple to write down, are separated by $O(J)+O(U)$ gaps from the remaining four zero-energy ground states. The existence of a gap for spin excitations means that the transport Green's function, Eq. 3, decays exponentially in the $\tau$ direction. Physically this may be interpreted 19] as the expected one-dimensional Anderson localization which is driven by backscattering among the two counterpropagating edge states.

Surprisingly, the problem of more than two sites can be solved exactly in the $U \gg J$ limit. To see this we consider the $\uparrow$-Hilbert subspace with just two states per site which may be represented as

$$
\begin{aligned}
b_{\uparrow}^{\dagger}|0\rangle & \rightarrow|0\rangle \\
c_{\uparrow}^{\dagger}|0\rangle & \rightarrow f^{\dagger}|0\rangle,
\end{aligned}
$$

where the f's correspond to spinless fermions. States with more than one fermion per site are forbidden by the Pauli exclusion principle; those with more than one boson per site are disfavored energetically by $H_{\eta}$ and the first term in $H_{J}$, Eq. 26. The elimination of the (zero in energy) vacuum state is justified a posteriori as the ground state energy will turn out to be negative; see Eq. 12 below. The different operators appearing in the Hamiltonian (Eq. 26) projected onto the subspace spanned by $|0\rangle$ and $f^{\dagger}|0\rangle$ can be represented solely in terms of the new fermion operators,

$$
\begin{array}{ll}
b_{\uparrow}^{\dagger} b_{\uparrow} \rightarrow 1-f^{\dagger} f & b_{\uparrow}^{\dagger} c_{\uparrow} \rightarrow f \\
c_{\uparrow}^{\dagger} c_{\uparrow} \rightarrow f^{\dagger} f & c_{\uparrow}^{\dagger} b_{\uparrow} \rightarrow f^{\dagger}
\end{array}
$$

This transformation is the analog of the Jordan-Wigner transformation [18] which maps the $\mathrm{x}-\mathrm{y}$ spin chain into a system of non-interacting tight-binding fermions. In this representation the Hamiltonian is quadratic, and setting $J=1$ we obtain: 


$$
H_{J}=-\sum_{n=1}^{L}\left(1+(-1)^{n} R\right)\left[f_{n}^{\dagger} f_{n}+f_{n+1}^{\dagger} f_{n+1}+(-1)^{n}\left(f_{n}^{\dagger} f_{n+1}^{\dagger}+f_{n} f_{n+1}\right)\right] .
$$

The Hamiltonian is now easily diagonalized. Due to the alternating term in Eq. 39 it is convenient to introduce separate fermion operators for even and odd sites,

$$
\begin{aligned}
f_{2 n} & =d_{n} \\
f_{2 n-1}^{\dagger} & =e_{n},
\end{aligned}
$$

where on the odd sites we have performed yet another particle-hole transformation. With this substitution, and upon Fourier transforming to $k$-space, the Hamiltonian may be rewritten as:

$$
H_{J}=\sum_{k}\left[2 d_{k}^{\dagger} d_{k}-2 e_{k}^{\dagger} e_{k}+(1-R)\left(d_{k}^{\dagger} e_{k}-e_{k}^{\dagger} d_{k}\right)+(1+R)\left(e^{i k} d_{k}^{\dagger} e_{k}-e^{-i k} e_{k}^{\dagger} d_{k}\right)\right] .
$$

Diagonalization is now a simple matter of computing the eigenvalues of a $2 \times 2$ non-Hermitian matrix, and we obtain:

$$
\epsilon_{k}= \pm 2 \sqrt{1-R^{2}}|\sin (k / 2)| .
$$

The eigenvectors are easily found. The $k=0$ piece of the Hamiltonian is defective, and once again the defect is lifted with the introduction of small negative $\mu_{c}$.

The ground state is constructed by filling up all of the negative energy states with the spinless fermions in the reduced Brillouin zone $0 \leq k<\pi$. Two things are remarkable about the dispersion relation: First, no gap opens up for $R \neq 0$. The expected spin gap at non-zero $R$ cannot be recovered in the $U \gg J$ limit because none of the excitations in the reduced Hilbert space carry spin. Second, the dispersion at the left and right Fermi points is linear, unlike the quadratic dispersion found for the ferromagnetic supersymmetric chain which describes the chiral metal [14. The linear dispersion is important to recover here because in the original plateau transition problem, unlike the chiral metal, the two spatial directions are equivalent.

In Hermitian supersymmetric theories the ground state is unique and has zero energy. Excited states pair into sectors of even and odd numbers of fermions and consequently the partition function $Z \equiv \operatorname{STr} e^{-\beta H_{\text {SUSY }}}=1$ at all temperatures $1 / \beta$. Here the supertrace operator $\mathrm{STr}$ is simply the ordinary trace over states with the insertion of an additional operator $(-1)^{N_{c}}$, where $N_{c}$ is the total fermion number operator introduced above. The operator $(-1)^{N_{c}}$ compensates for the anti-periodic boundary condition imposed on Grassman fields in the $\tau$-direction of the corresponding path-integral 14].

As the ground state energy is negative for the non-Hermitian Hamiltonian considered here, it is important to clarify how supersymmetry is manifest. In particular, at non-zero temperature (corresponding in the original network model to finite spatial extent and periodic boundary conditions in the y-direction) it is important to check whether the partition function really equals unity, $Z=1$. We first note that each term in the Hamiltonian $H_{\mathrm{SUSY}}=H_{U}+H_{J}+H_{\eta}$ either does not change the total fermion number, or changes it by an even integer. Therefore the many-body Hilbert space splits into two separate sectors: one with an odd number of fermions and $(-1)^{N_{c}}=-1$, the other with an even number and $(-1)^{N_{c}}=1$. The map between these two sectors is realized by fermion-valued supersymmetric charges,

$$
\begin{aligned}
Q_{1} & \equiv \sum_{n}\left[b_{\uparrow n}^{\dagger} c_{\uparrow n}-(-1)^{n} c_{\uparrow n}^{\dagger} b_{\uparrow n}\right] . \\
Q_{2} & \equiv \sum_{n}\left[(-1)^{n} b_{\uparrow n}^{\dagger} c_{\uparrow n}+c_{\uparrow n}^{\dagger} b_{\uparrow n}\right] .
\end{aligned}
$$

which are the analogues of those found for the supersymmetric ferromagnet [14].

Straightforward algebra confirms that $\left[H_{\mathrm{SUSY}}, Q_{1}\right]=\left[H_{\mathrm{SUSY}}, Q_{2}\right]=0$ in the $\mu_{c} \rightarrow 0$ limit, and also that $\left\{Q_{1}, Q_{2}\right\}=$ 0 . This holds true for $H_{\mathrm{SUSY}}$ in the finite- $U$ (Eq. 21) and in the infinite- $U$ (Eq. 26) limits. Therefore the charges $Q_{1}$ and $Q_{2}$ map one eigenstate with an odd number of fermions into a second state, degenerate with the first, but containing an even number of fermions. For example, two of the four degenerate eigen-states of the two-site problem, Eq. 30, have a single fermion while the other two states, Eq. 32, have a mixture of zero and two fermions. In this case we find: $Q_{1}\left|\Psi^{R}(1)\right\rangle=Q_{1}\left|\Psi^{R}(2)\right\rangle \propto\left(\left|\Psi^{R}(3+)\right\rangle-\left|\Psi^{R}(3-)\right\rangle\right)$. Operating with the charges a second time, on states $\left|\Psi^{R}(3 \pm)\right\rangle$, then returns states $\left|\Psi^{R}(1)\right\rangle$ and $\left|\Psi^{R}(2)\right\rangle$. In this way states which differ in content by one fermion are paired and also grouped into degenerate quartets. The only exception is the unique, zero-energy, empty vacuum state, $|0\rangle$, which is annihilated by both supersymmetric charges, $Q_{1}|0\rangle=Q_{2}|0\rangle=0$. All the other states (with either 
positive or negative energy) pair off and $Z=1$ at any temperature as expected from the supersymmetry of the construction. For the remainder of this paper, however, we consider only zero-temperature properties.

\section{DMRG CALCULATION}

To make further progress in the physically relevant finite- $U$ case we employ the Density Matrix Renormalization Group (DMRG) introduced by White [20]. We utilize the simpler and faster "infinite size" algorithm. It permits accurate evaluation of observables in the thermodynamic limit of large chain length but is less accurate for finite size chains.

As the Hamiltonian is non-Hermitian, both right and left eigenstates must be found. We employ the Davidson-Liu algorithm [21] extended by Morgan [22 to non-symmetric matrices. Sixteen on-site operators $S_{n}^{a}$, with $a=1,2, \cdots, 16$, are introduced. In normal-ordered form these are:

$$
\begin{array}{llll}
S^{1} \equiv b_{\uparrow}^{\dagger} b_{\uparrow} & S^{3} \equiv b_{\uparrow}^{\dagger} b_{\downarrow} & S^{9} \equiv b_{\uparrow}^{\dagger} c_{\uparrow} & S^{13} \equiv b_{\uparrow}^{\dagger} c_{\downarrow} \\
S^{4} \equiv \bar{b}_{\downarrow} b_{\uparrow} & S^{2} \equiv \bar{b}_{\downarrow} b_{\downarrow}+1 & S^{15} \equiv \bar{b}_{\downarrow} c_{\uparrow} & S^{11} \equiv \bar{b}_{\downarrow} c_{\downarrow} \\
S^{10} \equiv c_{\uparrow}^{\dagger} b_{\uparrow} & S^{16} \equiv c_{\uparrow}^{\dagger} b_{\downarrow} & S^{5} \equiv c_{\uparrow}^{\dagger} c_{\uparrow} & S^{7} \equiv c_{\uparrow}^{\dagger} c_{\downarrow} \\
S^{14} \equiv c_{\downarrow}^{\dagger} b_{\uparrow} & S^{12} \equiv c_{\downarrow}^{\dagger} b_{\downarrow} & S^{8} \equiv c_{\downarrow}^{\dagger} c_{\uparrow} & S^{6} \equiv c_{\downarrow}^{\dagger} c_{\downarrow}-1 .
\end{array}
$$

After the canonical transformation (Eq. 24) the exchange part of the supersymmetric Hamiltonian, Eq. 21, can be represented in terms of these on-site operators as:

$$
H_{J}=-\sum_{n=1}^{L} J_{n}\left[\sum_{a=1}^{8} \lambda_{a} S_{n}^{a} S_{n+1}^{a}+(-1)^{n} \sum_{a=9}^{16} \lambda_{a} S_{n}^{a} S_{n+1}^{a}\right]
$$

with coefficients $\lambda_{a}=-1$ for $a=1,2,10,11,14,15$ and otherwise $\lambda_{a}=+1$. The second term in the above equation is non-symmetric; it is composed of the eight operators $\left(S^{a}, a=9, \cdots, 16\right)$ which have fermionic character. When represented in terms of matrices with real-valued entries, the fermionic nature of these eight on-site operators leads to additional (-) signs, which have been taken into account in writing Eq. 45. In particular, these (-) signs are dictated by the fermion ordering convention along the chain which we adopt to define the many-body basis states: $c_{i}^{\dagger} \cdots c_{j}^{\dagger} \cdots c_{k}^{\dagger} \cdots|0\rangle$ with $i<j<k$. As the Hamiltonian commutes with the number operator on each site, $\mathcal{N}_{n} \equiv$ $\mathcal{N}_{\uparrow n}^{c}+\mathcal{N}_{\downarrow n}^{c}+\mathcal{N}_{\uparrow n}^{b}+\mathcal{N}_{\downarrow n}^{b}$, the on-site operators $S_{n}^{a}$ may be represented as $4 \times 4$ matrices acting on the 4 -dimensional single-site Hilbert space

$$
\begin{array}{ll}
|1\rangle \equiv b_{\uparrow}^{\dagger}|0\rangle & |3\rangle \equiv c_{\uparrow}^{\dagger}|0\rangle \\
|2\rangle \equiv \bar{b}_{\downarrow}|0\rangle & |4\rangle \equiv c_{\downarrow}^{\dagger}|0\rangle .
\end{array}
$$

For example, the only non-zero matrix element of $S^{1}$ is $\left\langle 1\left|S^{1}\right| 1\right\rangle=1$. Note that the state $\langle 2|=\langle 0|\left(\bar{b}_{\downarrow}\right)^{\dagger}=-\langle 0| b_{\downarrow}$ but the $(-)$ sign may be dropped as it always appears in pairs in the exchange Hamiltonian Eq. 45. We note that down spins are energetically disfavored due to the \pm 1 terms appearing in the definition of operators $S^{2}$ and $S^{6}$. The constant terms originate in the canonical transformation, Eq. 24.

As in the analytically tractable $U \gg J$ limit, the ground state remains four-fold degenerate in the $\mu_{c} \rightarrow 0$ limit even down to $U=0$. This is accounted for by the existence of two supersymmetric charges, Eq. 43, that commute with the Hamiltonian. The ground state expectation values of observables $\mathcal{O}$, which correspond to thermal expectations in the $\beta \rightarrow \infty$ limit, are computed using the supertrace formula,

$$
\langle\mathcal{O}\rangle \equiv \sum_{a=1}^{4}\left\langle\Psi^{L}(a)\left|(-1)^{N_{c}} \mathcal{O}\right| \Psi^{R}(a)\right\rangle
$$

\footnotetext{
${ }^{1}$ Note that $H \neq Q^{\dagger} Q$ as it would for a Hermitian supersymmetric field theory; hence, negative energy states are permitted.
} 
That both left and right eigenstates appear in Eq. 47 has its origin in the "resolution of identity" which is inserted into the partition function for the purpose of computing expectation values:

$$
1=\sum_{a}\left|\Psi^{R}(a)\right\rangle\left\langle\Psi^{L}(a)\right|
$$

here the sum runs over the entire Hilbert space. (The states span the Hilbert space because the defect was eliminated via the introduction of $\mu_{c}<0$.)

We introduce reduced density matrices for both of the augmented blocks, each of Hilbert space size $4 M$, by computing partial traces over states on the right (or left) halves of the chain. The reduced density matrix for the left half of the chain is given by:

$$
\rho_{i j}=\frac{1}{8} \sum_{a=1}^{4} \sum_{i^{\prime}=1}^{4 M}\left[\frac{\Psi_{i i^{\prime}}^{R}(a) \Psi_{j i^{\prime}}^{R}(a)}{\left\langle\Psi^{R}(a) \mid \Psi^{R}(a)\right\rangle}+\frac{\Psi_{i i^{\prime}}^{L}(a) \Psi_{j i^{\prime}}^{L}(a)}{\left\langle\Psi^{L}(a) \mid \Psi^{L}(a)\right\rangle}\right] ;
$$

a similar formula defines the density matrix for the right half of the chain. Here $\Psi_{i i^{\prime}}^{L, R} \equiv\left\langle i, i^{\prime} \mid \Psi^{L, R}\right\rangle$ are the matrix elements of the many-body wavefunction projected onto a basis of states labeled by unprimed roman index $i$ which covers the left half of the chain and primed index $i^{\prime}$ which covers the right half. The states are normalized in Eq. 49 to insure that $\operatorname{Tr} \rho=\sum_{i} \rho_{i i}=1$. As can be verified numerically, all of the eigenvalues of $\rho$ are real (as it is a symmetric matrix) and, more significantly, they are positive and hence may be interpreted as probabilities. The DMRG truncation procedure thus corresponds to the elimination of the least-important sectors of the Hilbert space. Our choice for the reduced density matrix agrees with that of Bursill, Xiang, and Gehring [23] but differs from that of Nishino 24] who argues that a product of left with right eigenstates is optimal in a variational sense. However, the resulting reduced density matrix is non-Hermitian and some of its eigenvalues are negative; they cannot be interpreted as probabilities. Further, it is a difficult numerical problem to find a large fraction of the eigenstates of a non-Hermitian matrix. Since the reduced density matrix is introduced solely for the purpose of truncating, in a systematic manner, the rapid growth of the Hilbert space with chain length, here we adopt the symmetric choice (Eq. 49) and check that observables do not change significantly when the size $M$ of the block's Hilbert space is enlarged.

The exactly solvable $U \gg J$ limit permits another important check on the quality of the DMRG algorithm. We have verified that the DMRG procedure accurately reproduces the energies of the four degenerate ground states obtained from the dispersion relation (Eq. 42) as the chain grows. For example, in the case $U=10 \mathrm{~J}$ and for a block Hilbert space size of $M=80$, the energies agree to within 1 part in $10^{4}$ at chain lengths up through $L=16$.

We continue to lift the degeneracy, and the defectiveness, of the Hamiltonian via a small chemical potential for fermions, Eq. 31. Typically we set $\mu_{c}=-1.0 \times 10^{-6}$ in units where $J=1$. Although each of the four terms in Eq. 47 in general depends strongly on $\mu_{c}$ we have verified that this dependence drops out in the sum over the four nearly degenerate ground states (see Fig. 3). The rest of the DMRG algorithm is standard: During each iteration, the chain is cut into left and right pieces, the reduced density matrix for each half is formed and diagonalized, new representations of the operators are found, and finally two sites are added to the middle of the chain and the resulting new Hamiltonian is diagonalized via the modified Davidson-Liu algorithm. The near degeneracy and defectiveness of $H_{J}$ slows down the diagonalization considerably and can be partly compensated by increasing the size of the Davidson subspace. We run the double-precision, vectorized, multi-processor C-code on a Cray EL-98 computer. The largest computations take about 1 Gbyte of memory and require several weeks to run.

\section{DMRG RESULTS}

Previous work on the replica limit of the Heisenberg [7] and Hubbard [8] models focused on the scaling of the spin gap $\Delta_{s}(R)$ as a function of $R$, the staggered coupling between neighboring sites. It is difficult to gain direct access to the spin gap in the supersymmetric chain due to the plethora of low-lying excitations in the pure spin-up sector (discussed in Sec. V) which persist at finite- $U$. To isolate spin-flip excitations would require finding many low-lying eigenvectors, a problem for which the DMRG method is not well suited [23].

Critical behavior is evident, however, in the expectation value of a spin-flip operator which lives on the bonds between neighboring sites. It is important to note though that according to Eq. 47 supersymmetry-invariant operators (those which commute with the supersymmetric charges $Q_{1,2}$ ), have zero expectation value. For example, $\left\langle H_{\mathrm{SUSY}}\right\rangle=$ 0 despite the fact that the ground state energy is negative. The non-supersymmetric fermion spin-flip operator $(-1)^{N_{c}} S_{n}^{7} S_{n+1}^{7}$, on the other hand, is an appropriate choice. Even with no applied dimerization $(R=0)$, its groundstate expectation value 


$$
\left\langle(-1)^{N_{c}} S_{n}^{7} S_{n+1}^{7}\right\rangle=\left\langle(-1)^{N_{c}} c_{\uparrow n}^{\dagger} c_{\downarrow n} c_{\uparrow n+1}^{\dagger} c_{\downarrow n+1}\right\rangle \equiv B(n, n+1)
$$

has even/odd modulation due to the free boundary condition at the chain ends. This modulation can be isolated by defining

$$
\Delta(n) \equiv(-1)^{n}[B(n, n+1)-B(n-1, n)]+c . c .,
$$

and it can be understood as follows: chains with an even number of sites and free boundary conditions at the chain ends have no reflection symmetry about any site center. The chain ends induce slight dimerization (which also breaks reflection symmetry about site centers). Each time the chain length is increased by two sites during a DMRG iteration the pattern of weak and strong bonds alternates; the term $(-1)^{n}$ appearing in Eq. 51 accounts for this alternation.

As $\Delta(n)$ is the expectation value of an operator which induces dimerization at $R \neq 0$ it has scaling dimension $x_{\Delta}$ which is related to the correlation length exponent by the standard scaling relation: $\nu=\left(2-x_{\Delta}\right)^{-1}$ [25]. Therefore, in a finite system we expect

$$
\Delta(n) \sim L^{-x_{\Delta}}, \quad L \rightarrow \infty
$$

This observation provides us with a means of extracting $x_{\Delta}$ from the DMRG by measuring the expectation value of the dimerization operator on the central bonds, $\Delta(L / 2)$, at each DMRG step.

To test this method, and the DMRG algorithm, we first examine the ordinary isotropic, nearest-neighbor, spin- $1 / 2$ $\mathrm{SU}(2)$ quantum antiferromagnet. In this case the dimension of the dimerization operator $(-1)^{n} \vec{S}_{n} \cdot \vec{S}_{n+1}$ is known exactly [26]: $x_{\Delta}=1 / 2$. For chains up to length $L=204$ and block Hilbert space size $M=256$ we find results consistent with the theoretical prediction. Namely, if we calculate $x_{\Delta}$ by fitting the log-log plot in Fig. 2 to a linear function, for points $L>10$, we find $x_{\Delta} \approx 0.6$. The discrepancy with the exact result we attribute to the presence of a marginally irrelevant operator [26], and other less relevant scaling operators. These corrections to scaling, which lead to a slightly larger value of the effective exponent, are evidenced by a slight curvature of the plot in Fig. 2 . More precisely, the marginally irrelevant operator makes multiplicative logarithmic corrections to scaling [25]. We take these corrections into account by fitting the values to the functional form $\Delta(L / 2)=L^{-x_{\Delta}}(\log (L))^{\gamma} f\left(L^{-1}\right)$. The function $f(x)$ is assumed analytic around the origin and we expand it via Taylor series, keeping only the first two terms, $f(x)=C_{1}+C_{2} x$. With this procedure we obtain $x_{\Delta} \approx 0.5$ and $\gamma \approx 0.5$, in agreement with theoretical expectations.

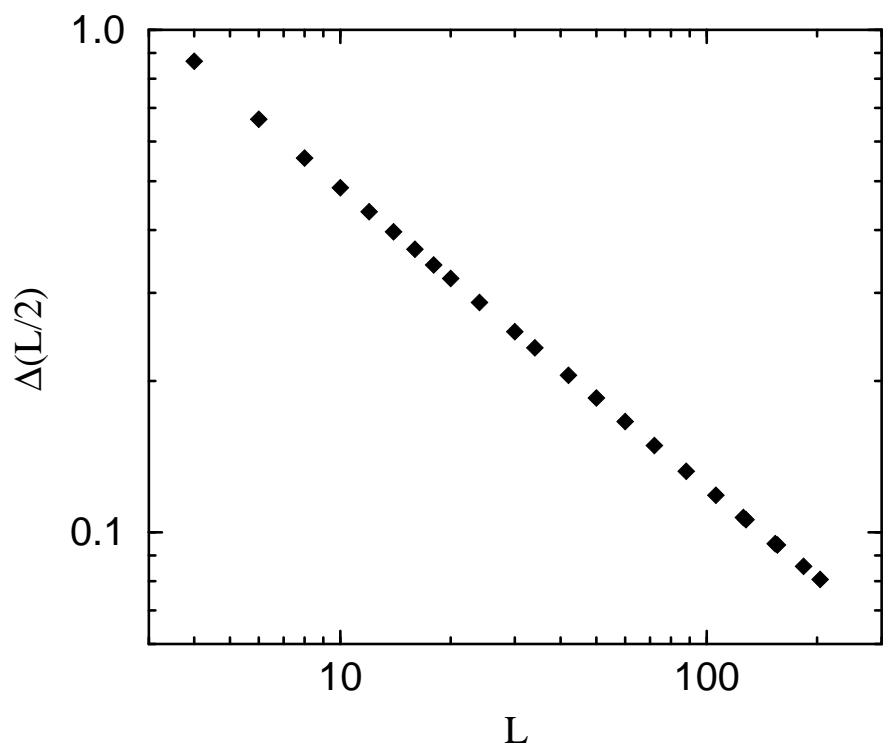

FIG. 2. Expectation value of the dimerization operator on the central bonds, $\Delta(L / 2)$, for the $S=1 / 2$ Heisenberg spin chain; $L$ is the chain length. The infinite-size DMRG method with a block Hilbert space size of $M=256$ is used. Slight curvature of the plot signals the presence of other less relevant scaling operators and logarithmic corrections to scaling.

Returning to the supersymmetric chain, scaling is evident in Fig. 3 where $x_{\Delta} \approx 1.8$ is calculated by fitting the $M=160$ points with $L>10$ to a straight line; curvature in the plot signals once again corrections to scaling. We 
account for these less relevant operators by fitting $\Delta(L / 2)$ to the functional form $\Delta(L / 2)=L^{-x_{\Delta}} f\left(L^{-1}\right)$. As in the case of the $S U(2)$ Heisenberg chain above, we Taylor-expand $f(x)=C_{1}+C_{2} x+\cdots$, keeping only the first two terms. Following this procedure we obtain $x_{\Delta}=1.58 \pm 0.07$. The error is estimated by choosing different sets of points to include in the fit; statistical errors are smaller, at the few percent level. This value of the scaling dimension is consistent with the more precise value $x_{\Delta}=2-\nu^{-1}=1.57 \pm 0.01$ (using $\nu=2.33 \pm 0.05$ ), reported by other groups [5].

The DMRG works best for phases with a gap to low-lying excitations; we do not expect, nor do we find, very good quantitative agreement at the critical point. To obtain more accurate scaling it would be necessary to employ the "finite-size" DMRG algorithm and carry out careful finite-size analysis by varying both the chain length $L$ and the dimerization parameter $R$ to search for a universal scaling function.

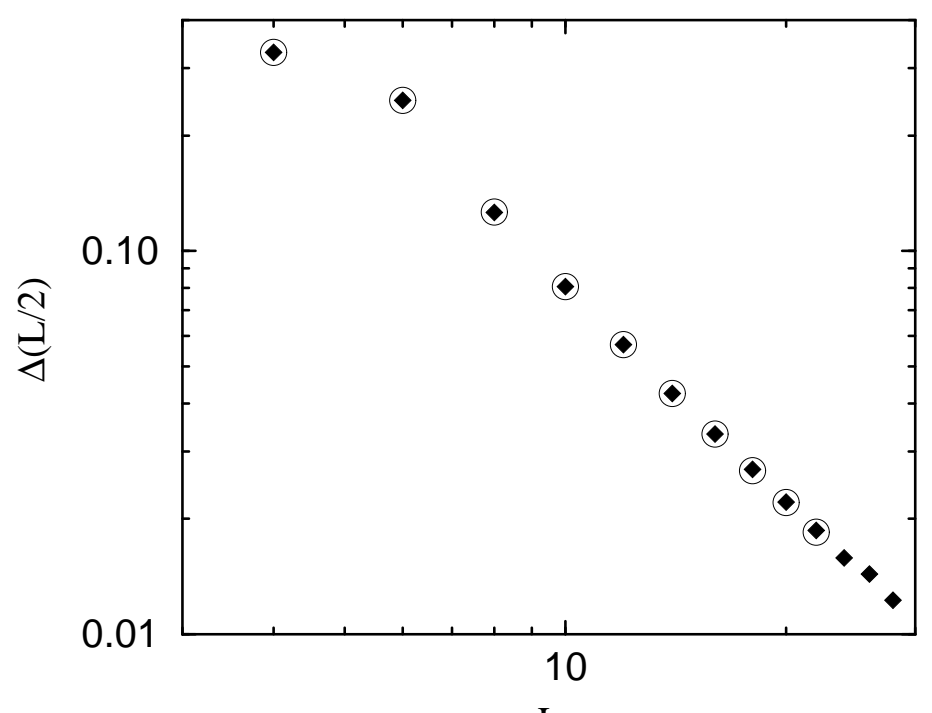

FIG. 3. Expectation value of the dimerization operator on the central bonds, $\Delta(L / 2)$, versus system length $L$ for $U=0$ and at the critical point $R=0$ of the SUSY chain. Results for two different runs are shown: filled diamonds are for a block Hilbert space size of $M=160$ [corresponding to a total Hilbert space size of $(4 M)^{2}=409,600$ ] and $\mu_{c}=-10^{-6}$ in units where $J=1$. Open circles are for a more accurate calculation at $M=256$ [corresponding to a total Hilbert space size of 1,048,576] and $\mu_{c}=-10^{-4}$. The two calculations agree precisely up through chain length $L=8$. For $L>8$ the entire Hilbert space cannot be accommodated; instead it is truncated via the DMRG algorithm. Nevertheless, the two calculations continue to agree closely out through chain length $L=22$, the largest chain length reached in the $M=256$ calculation. At $L=26$ the $M=160$ point deviates from the expected scaling form. We attribute the breakdown of scaling at large chain length to the truncation of the Hilbert space.

\section{DISCUSSION}

What have we learned from the above analysis? First, the scaling in Fig. 3 demonstrates the utility of the supersymmetric approach to understanding transport at the IQHE plateau transition. The series of transformations outlined above which map the network model described by Eq. 1 to the pure, but rather complicated, supersymmetric spin-chain Hamiltonian Eq. 45 appears to work. The replica trick is avoided and we obtain instead a concrete model amenable to analytical and numerical study. Deep inside a plateau $(R \rightarrow \pm 1)$ the chain breaks up into disconnected two-site systems and Anderson localization in the interior is complete. At $R=-1$ the two spins at the chain ends are fully polarized in the $\uparrow$-direction and no extended edge currents flow; in the opposite $R=+1$ limit the two spins are free and the conductivity increases by a quantum [27].

Second, and more importantly, the Hamiltonian is analytically tractable when projected onto the Hilbert space with only one type $(\uparrow)$ of boson and fermion. This is the $U \gg J$ limit, Eq. 25, and there is the intriguing possibility that the Hamiltonian can be studied systematically down to the physical regime of finite $U$. This approach differs fundamentally from previous weak-coupling studies [9] where runaway RG flows toward strong coupling in the generic IQHE case suggested the existence of a strong-coupling fixed point. The starting point here, by contrast, would be at 
very strong coupling. It may then be possible to incorporate spin excitations perturbatively about the strong coupling limit.

A number of unanswered questions remain. The significance of the defectiveness of the Hamiltonian is unclear. It is a robust feature, as it occurs at both infinite and finite $U$. Also unanswered is the question of whether or not it will be possible to construct a two-dimensional conformal field theory (CFT) of the critical point. As pointed out by Mudry et al. [28] a candidate CFT would have to be non-unitary, thus allowing for scaling dimensions not predicted by the Kac determinant. This opens up the possibility of identifying a CFT that contains an operator with a scaling dimension $x_{\Delta} \approx 11 / 7$ in its spectrum; such an operator, if associated with a perturbation that induces a flow from the critical point to the non-critical phases of two adjacent Hall plateaus, would give rise to the expected correlation length exponent $\nu=\left(2-x_{\Delta}\right)^{-1} \approx 7 / 3$. Searching for such a field theory among CFT's which are also supersymmetric has not been successful [29]. We believe that further studies of the supersymmetry approach to the Chalker-Coddington network model will shed more light on this matter.

\section{ACKNOWLEDGMENTS}

We thank L. Balents, R. Bursill, D. Nelson, N. Read, D. Serban, R. Shankar, and X.-G. Wen for helpful discussions, and W. Becker for pointing out references on the diagonalization of large matrices. Some of our work was done at the Aspen Center for Physics. Computations were carried out at the Theoretical Physics Computing Facility at Brown University. This work was supported in part by the National Science Foundation through Grants Nos. DMR-9313856 and No. DMR-9357613 and by a grant from the the Alfred P. Sloan Foundation (J.B.M.).

[1] H. Levine, S. B. Libby, and A. M. M. Pruisken, Phys. Rev. Lett. 51, 1915 (1983); A. M. M. Pruisken in The Quantum Hall Effect edited by R. E. Prange and S. M. Girvin (Springer-Verlag, New York, 1990).

[2] H. P. Wei, D. C. Tsui, M. A. Paalanen, and A. M. M. Pruisken, Phys. Rev. Lett. 61, 1294 (1988).

[3] S. Koch, R. J. Haug, K. von Klitzing and K. Ploog, Phys. Rev. Lett. 67, 883 (1993).

[4] J. T. Chalker and P. D. Coddington, J. Phys. C 21, 2665 (1988).

[5] D.-H. Lee, Z. Wang, and S. A. Kivelson, Phys. Rev. Lett. 70, 4130 (1993); D. K. K. Lee and J. T. Chalker, Phys. Rev. Lett. 72, 1510 (1994); Z. Q. Wang, D.-H. Lee, and X.-G. Wen, Phys. Rev. Lett. 72, 2454 (1994); V. Kagalovsky, B. Horovitz, and Y. Avishai, Phys. Rev. B 52, 17044 (1995); J. T. Chalker and A. Dohmen, Phys. Rev. Lett. 75, 4496 (1995).

[6] G.V. Milnikov amd I.M. Sokolov, JETP Lett. 48, 536 (1988).

[7] Dung-Hai Lee, Phys. Rev. B 50, 10,788 (1994).

[8] Dung-Hai Lee and Ziqiang Wang, Phil. Mag. Lett. 73, 145 (1996).

[9] A. W. W. Ludwig, M. P. A. Fisher, R. Shankar, and G. Grinstein, Phys. Rev. B 50, 7526 (1994).

[10] A. McKane, Phys. Lett. 76A, 22 (1980).

[11] K. B. Efetov, Adv. Phys. 32, 53 (1983).

[12] Ian Affleck, J. Phys. C 17, 2323 (1984).

[13] M. R. Zirnbauer, Annalen der Physik 3, 513 (1994).

[14] L. Balents, M. P. A. Fisher, and M. Zirnbauer, Nucl. Phys. B483, 601 (1997).

[15] Ilya A. Gruzberg, N. Read, and Subir Sachdev, "Scaling and Crossover Functions for the Conductance in the Directed Network Model of Edge States", cond-mat/9612038.

[16] S.A. Trugman, Phys. Rev. B 27, 7539 (1983).

[17] A.J. McKane and M. Stone, Ann. Phys. 131, 36 (1981).

[18] J.W. Negele and H. Orland, Quantum Many-Particle Systems (Addison-Wesley, Redwood City, 1988).

[19] Leon Balents, private communication.

[20] S. R. White, Phys. Rev. Lett. 69, 2863 (1992); Phys. Rev. B 48, 10,345 (1993).

[21] Ernest R. Davidson, J. Comp. Phys. 17, 87 (1975); Comp. Phys. 7, 519 (1993).

[22] Ronald B. Morgan, J. Comp. Phys. 101, 287 (1992).

[23] R. J. Bursill, T. Xiang, and G. A. Gehring, "Density Matrix Renormalization Group for a Quantum Spin Chain at Non-Zero Temperature," cond-mat/9609001.

[24] Tomotoshi Nishino, J. Phys. Soc. Japan 64, 3598 (1995); T. Nishino and K. Okunishi, "Density Matrix and Renormalization for Classical Lattice Models," cond-mat/9610107.

[25] J. Cardy, Scaling and Renormalization in Statistical Physics (Cambridge University Press, Cambridge 1996).

[26] I. Affleck in Fields, Strings, and Critical Phenomena, E. Brezin and J. Zinn-Justin eds. (North-Holland, Amsterdam 1990). 
[27] Yong Baek Kim, "Edge States of Integral Quantum Hall States Versus Edge States of Antiferromagnetic Quantum Spin Chains," cond-mat/9601050.

[28] C. Mudry, C. Chamon, and X.-G. Wen, Nucl. Phys. B466, 383 (1996).

[29] X.-G. Wen, private communication. 